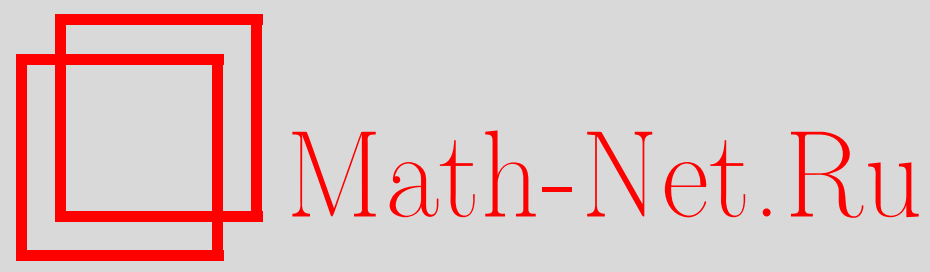

Е. Е. Тыртышников, В. Н. Чугунов, О задачах окаймления и модификации эрмитовых матриц, Матем. заметки, 2002, том 71, выпуск 1, 130-134

DOI: https://doi.org/10.4213/mzm334

Использование Общероссийского математического портала Math-Net.Ru подразумевает, что вы прочитали и согласны с пользовательским соглашением http://www.mathnet.ru/rus/agreement

Параметры загрузки:

IP : 54.157 .27 .8

26 апреля 2023 г., $17: 42: 44$

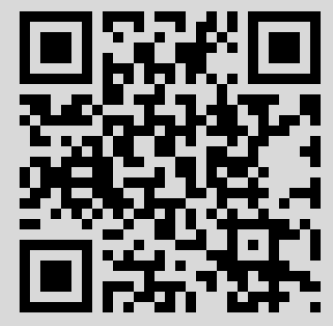




\title{
О ЗАДАЧАХ ОКАЙМЛЕНИЯ И МОДИФИКАЦИИ ЭРМИТОВЫХ МАТРИЦ
}

\author{
Е. Е. Тыртышников, В.Н. Чугунов
}

Получены необходимые и достаточные условия разрешимости задач окаймления и модификации порядка $r$ эрмитовых матриц. В задаче окаймления требуется по предписанным собственным значениям построить эрмитову $((n+r) \times(n+r))$-матрицу с заданньм $(n \times n)$-блоком $A_{11}$ в блочном $(2 \times 2)$-представлении. Задача модификации заключается в построении эрмитовой $(n \times n)$-матрицы $B$ ранга не более $r$ такой, чтобы матрица, полученная при сложении матрицы $B$ с заданной эрмитовой $(n \times n)$-матрицей $A$, имела требуемый спектр. Приведена оценка для минимального числа различных собственных значений у решений данных задач.

Библиограффия: 2 названия.

1. Введение. Пусть $A$ - эрмитова $(n \times n)$-матрица с собственньми значениями $\lambda_{1}, \lambda_{2}, \ldots, \lambda_{n}\left(\lambda_{1} \leqslant \lambda_{2} \leqslant \cdots \leqslant \lambda_{n}\right)$.

Под задачей окаймления порядка $r$ матрицы $A$ (задача I) понимается процесс построения эрмитовой $((n+r) \times(n+r))$-матрищы $B$ с предписанными собственными значениями $\widehat{\lambda}_{1}, \widehat{\lambda}_{2}, \ldots, \hat{\lambda}_{n+r}\left(\hat{\lambda}_{1} \leqslant \widehat{\lambda}_{2} \leqslant \cdots \leqslant \widehat{\lambda}_{n+r}\right)$ такой, что

$$
(B)_{i j}=(A)_{i j}, \quad 1 \leqslant i, j \leqslant n .
$$

В задаче модификации ранга $r$ матрищы $A$ (задача II) требуется построить эрмитову $(n \times n)$-матрицу $B$ ранга не более $r$ такую, что матрица $(A+B)$ имеет заданный спектр $\hat{\lambda}_{1}, \widehat{\lambda}_{2}, \ldots, \hat{\lambda}_{n}\left(\widehat{\lambda}_{1} \leqslant \widehat{\lambda}_{2} \leqslant \cdots \leqslant \widehat{\lambda}_{n}\right)$.

Решение этих проблем для $r=1$ дают следуюшие теоремы.

Теорема 1. Задача окаймления разрешима тогда и только тогда, когда

$$
\widehat{\lambda}_{1} \leqslant \lambda_{1} \leqslant \widehat{\lambda}_{2} \leqslant \lambda_{2} \leqslant \cdots \leqslant \widehat{\lambda}_{n} \leqslant \lambda_{n} \leqslant \widehat{\lambda}_{n+1}
$$

Работа выполнена при финансовой поддержке Российского фонда фундаментальных исследований, грант № 99-01-00017. 
ТЕорема 2. Для существования решения задачи модификаиии необходимо и достаточно выполнение следующих условий:

$$
\widehat{\lambda}_{i} \leqslant \lambda_{i+1} \leqslant \widehat{\lambda}_{i+2}, \quad \lambda_{i} \leqslant \widehat{\lambda}_{i+1} \leqslant \lambda_{i+2}, \quad i=1,2, \ldots, n-2 .
$$

Доказательство этих утверждений можно найти в [1], [2].

Цель данной заметки двоякая. С одной стороны, представить решение этих задач для произвольного $r$. Этому посвящен раздел 2. С другой стороны, мы хотим исследовать вопрос о том, как сильно можем распоряжаться спектром возмущенной матрищы, какова степень свободы. В разделе 3 приведены оценки для минимального числа различных собственных значений у решений данных задач.

\section{2. Теоремы разрешимости.}

2.1. Задача окаймления. В данном разделе будут сформулированы и обоснованы необходимые и достаточные условия существования решения задачи I.

ТЕОРема 3. Задача окаймления разрешима тогда и только тогда, когда имеют место неравенства

$$
\widehat{\lambda}_{i} \leqslant \lambda_{i} \leqslant \widehat{\lambda}_{i+r}, \quad i=1, \ldots, n .
$$

ДоказАтЕЛЬСтво. Необходимость. Обозначим через $A^{(k)}((n+k) \times(n+k))$-подматрицу $B$ в верхнем левом углу для $k=0,1, \ldots, r$, а через $\mu_{1}^{(k)}, \mu_{2}^{(k)}, \ldots, \mu_{n+k}^{(k)}-$ eе собственные значения. Тогда

$$
A^{(0)}=A, \quad A^{(r)}=B, \quad \mu_{i}^{(0)}=\lambda_{i}, \quad i=1, \ldots, n, \quad \mu_{i}^{(r)}=\widehat{\lambda}_{i}, \quad i=1, \ldots, n+r .
$$

Заметим, что каждая из матриц $A^{(j)}, j=1,2, \ldots, r$, является решением задачи окаймления порядка 1 для матрицы $A^{(j-1)}$. Поэтому по теореме 1

$$
\mu_{i}^{(j)} \leqslant \mu_{i}^{(j-1)} \leqslant \mu_{i+1}^{(j)}, \quad i=1, \ldots, n, \quad j=1, \ldots, r .
$$

Применяя формулу (2.2) и левое соотношение $(2.3)$ для $j=r, \ldots, 1$, получаем

$$
\widehat{\lambda}_{i}=\mu_{i}^{(r)} \leqslant \mu_{i}^{(r-1)} \leqslant \cdots \leqslant \mu_{i}^{(1)} \leqslant \mu_{i}^{(0)}=\lambda_{i}, \quad i=1, \ldots, n
$$

или

$$
\widehat{\lambda}_{i} \leqslant \lambda_{i}, \quad i=1, \ldots, n .
$$

Аналогично из (2.2) и правых неравенств (2.3) для $j=1, \ldots, r$ имеем

$$
\lambda_{i}=\mu_{i}^{(0)} \leqslant \mu_{i+1}^{(1)} \leqslant \mu_{i+2}^{(2)} \leqslant \cdots \leqslant \mu_{i+r}^{(i+r)}=\widehat{\lambda}_{i+r}, \quad i=1, \ldots, n,
$$

или

$$
\lambda_{i} \leqslant \widehat{\lambda}_{i+r}, \quad i=1, \ldots, n .
$$

Объединяя (2.4) и (2.5), приходим к условию (2.1). 
Достаточность докажем по индукции. При $r=1$ получаем теорему 1 . Для обоснования теоремы для $r>1$ построим набор чисел $\mu_{1}, \ldots, \mu_{n+r-1}$ таких, что

$$
\begin{gathered}
\widehat{\lambda}_{i} \leqslant \mu_{i} \leqslant \widehat{\lambda}_{i+1}, \quad i=1, \ldots, n+r-1, \\
\mu_{i} \leqslant \lambda_{i} \leqslant \mu_{i+r-1}, \quad i=1, \ldots, n .
\end{gathered}
$$

В качестве $\mu_{i}$ можно взять, например,

$$
\mu_{i}=\frac{c_{i}+d_{i}}{2}, \quad i=1, \ldots, n+r-1,
$$

где

$$
\begin{aligned}
c_{i} & = \begin{cases}\widehat{\lambda}_{i}, & i=1, \ldots, r-1, \\
\max \left(\widehat{\lambda}_{i}, \lambda_{i-(r-1)}\right), & i=r, \ldots, n+r-1,\end{cases} \\
d_{i} & = \begin{cases}\min \left(\widehat{\lambda}_{i+1}, \lambda_{i}\right), & i=1, \ldots, n, \\
\widehat{\lambda}_{i+1}, & i=n+1, \ldots, n+r-1 .\end{cases}
\end{aligned}
$$

Покажем справедливость (2.6) и (2.7). В силу (2.1) и соотношений $\lambda_{1} \leqslant \cdots \leqslant \lambda_{n}$, $\widehat{\lambda}_{1} \leqslant \cdots \leqslant \widehat{\lambda}_{n+r}$ заключаем, что $c_{i} \leqslant d_{i}$. Поэтому из условий $c_{i} \geqslant \widehat{\lambda}_{i}, d_{i} \leqslant \widehat{\lambda}_{i+1}$ следует (2.6), а из условия $d_{i} \leqslant \lambda_{i}$ - левая часть (2.7). Так как $c_{i} \geqslant \lambda_{i-(r-1)}$, то и $\mu_{i} \geqslant \lambda_{i-(r-1)}$. Заменяя в этом неравенстве $i$ на $i+r-1$, приходим к (2.7).

По индуктивному предположению для $(n \times n)$-матрицы $A$ и чисел $\mu_{1}, \ldots, \mu_{n+r-1}$ построим $((n+r-1) \times(n+r-1))$-матрицу $\widetilde{B}$ с собственными значениями $\mu_{1}, \ldots, \mu_{n+r-1}$ такую, что

$$
(\widetilde{B})_{i, j}=(A)_{i j}, \quad 1 \leqslant i, j \leqslant n .
$$

Это возможно в силу (2.7). Условия (2.6) позволяют окаймить матрицу $\widetilde{B}$ столбцом справа и строкой снизу так, чтобы итоговая $((n+r) \times(n+r))$-матрица имела заданный спектр $\widehat{\lambda}_{1}, \ldots, \widehat{\lambda}_{n+r}$. Теорема доказана.

\section{2. Задача модификации.}

ТЕорема 4. Задача модификации разрешима тогда и только тогда, когда

$$
\text { 1) } \hat{\lambda}_{i} \leqslant \lambda_{i+r} \leqslant \hat{\lambda}_{i+2 r}, \quad \text { 2) } \quad \lambda_{i} \leqslant \widehat{\lambda}_{i+r} \leqslant \lambda_{i+2 r}, \quad i=1, \ldots, n-2 r .
$$

ДокАЗАТЕЛЬСТво. Необходимость данного утверждения доказана в [2].

Достаточность проведем по аналогии с теоремой 3 по индукции. Для $r=1$ имеем теорему 2 . При $r>1$ выберем числа $\mu_{1}, \ldots, \mu_{n}$, удовлетворяющие условиям

$$
\mu_{i} \leqslant \widehat{\lambda}_{i+1} \leqslant \mu_{i+2}, \quad \widehat{\lambda}_{i} \leqslant \mu_{i+1} \leqslant \widehat{\lambda}_{i+2}, \quad i=1, \ldots, n-2
$$

И

$$
\lambda_{i} \leqslant \mu_{i+r-1} \leqslant \lambda_{i+2(r-1)}, \quad \mu_{i} \leqslant \lambda_{i+r-1} \leqslant \mu_{i+2(r-1)}, \quad i=1, \ldots, n-2(r-1) .
$$

Если положить

$$
\mu_{i}=\frac{c_{i}+d_{i}}{2}, \quad i=1, \ldots, n
$$


где

$$
\begin{gathered}
c_{i}= \begin{cases}\min \left(\widehat{\lambda}_{1}, \lambda_{1}\right), & i=1, \\
\widehat{\lambda}_{i-1}, & i=2, \ldots, r-1, \\
\max \left(\widehat{\lambda}_{i-1}, \lambda_{i-(r-1)}\right), & i=r, \ldots, n,\end{cases} \\
d_{i}= \begin{cases}\min \left(\widehat{\lambda}_{i+1}, \lambda_{i+r-1}\right), & i=1, \ldots, n-(r-1), \\
\hat{\lambda}_{i+1}, & i=n-r+2, \ldots, n-1, \\
\max \left(\widehat{\lambda}_{n}, \lambda_{n}\right), & i=n,\end{cases}
\end{gathered}
$$

то из соотношений $\lambda_{1} \leqslant \ldots \leqslant \lambda_{n}, \widehat{\lambda}_{1} \leqslant \ldots \widehat{\lambda}_{n}$ и условий $(2.8)$ несложно вьвести $(2.9)$ и $(2.10)$.

Благодаря неравенствам (2.10) построим по индуктивному предположению $(n \times n)$ матрицу $B_{1}$ ранга не более $r-1$ такую, что матрица $\widetilde{B}=A+B_{1}$ имеет собственные значения $\mu_{1}, \ldots, \mu_{n}$. Условие $(2.9)$ в силу теоремы 2 обеспечивает существование одноранговой матрищы $B_{2}$ такой, что спектр матрицы $\widetilde{B}+B_{2}$ составляют числа $\widehat{\lambda}_{1}, \ldots, \widehat{\lambda}_{n}$. Решение задачи модификации в данном случае представимо в виде матрицы $B=B_{1}+B_{2}$, ранг которой не более суммы рангов $B_{1}$ и $B_{2}$, т.е. не более $r$. Теорема доказана.

3. Оценки для задаваемого спектра. Изучим теперь вопрос о минимальном количестве различных чисел среди задаваемого спектра $\widehat{\lambda}_{1}, \ldots, \widehat{\lambda}_{n+r}$ (в задаче I) и $\widehat{\lambda}_{1}, \ldots$, $\widehat{\lambda}_{n}$ (в задаче II). В данном разделе считаем, что все собственные значения $A$ простые.

\section{1. Задача окаймления.}

ТЕОРемА 5. Минимальное число различных собственных значений среди задаваемых $\widehat{\lambda}_{1}, \ldots, \widehat{\lambda}_{n+r}$ в задаче окаймления не может быть меньше

$$
2+\left\lceil\frac{(n-r-2)_{+}}{r+1}\right\rceil,
$$

әде

$$
(x)_{+}= \begin{cases}x, & x \geqslant 0 \\ 0, & x<0\end{cases}
$$

ДокАЗАТЕЛЬСТво. Главными условиями, налагаемыми на числа $\widehat{\lambda}_{1}, \ldots, \hat{\lambda}_{n+r}$, являются соотношения

$$
\widehat{\lambda}_{i} \leqslant \lambda_{i} \leqslant \widehat{\lambda}_{i+r}, \quad i=1, \ldots, n .
$$

Уменьшить число различных $\widehat{\lambda}_{1}, \ldots, \widehat{\lambda}_{n+r}$ можно лишь за счет слияния некоторых из них. Из условия (3.2) очевидно, что точки $\lambda_{1}$ и $\lambda_{n}$ как границы спектра матрицы $A$ накладьвают условия на $\widehat{\lambda}_{1}$ и $\widehat{\lambda}_{n+r}$, не давая последним приблизиться. Поэтому $\lambda_{1}$ и $\lambda_{n}$ являются двумя граничными точками слияния. Несложно проверить, что, положив

$$
\widehat{\lambda}_{1}=\cdots=\widehat{\lambda}_{r+1}=\lambda_{1}, \quad \widehat{\lambda}_{n}=\cdots=\widehat{\lambda}_{n+r}=\lambda_{n}
$$

мы обеспечим вьполнение условий (3.2), однако при этом увеличение кратностей $\lambda_{1}$ и $\lambda_{n}$ невозможно. После такого выбора у нас остается $(n-r-2)$ чисел спектра $\widehat{\lambda}_{r+2}, \ldots$, $\widehat{\lambda}_{n-1}$, которыми мы еще можем распорядиться. 
Запишем (3.2) в виде

$$
\lambda_{i-r} \leqslant \widehat{\lambda}_{i} \leqslant \lambda_{i}
$$

и рассмотрим это неравенство для $i=k+r, k=2, \ldots, n-r-1$. Получим соотношения

$$
\lambda_{k} \leqslant \widehat{\lambda}_{k+r} \leqslant \lambda_{k+r}, \quad k=1, \ldots, n-r-1 .
$$

Отсюда следует, что каждое из чисел $\widehat{\lambda}_{k+r}$ должно принадлежать интервалу $\left[\lambda_{k}, \lambda_{k+r}\right]$, $k=2, \ldots, n-r-1$. Эти интервалы расположены последовательно, пересечение нескольких рядом стоящих не пусто, при этом максимальное число интервалов, имеющих общую точку, равно $r+1$. Разобъем все множество этих интервалов на группы по $r+1$ с последовательньми номерами (в последней может быть меньше). Так как в каждой группе существует точка, принадлежащая всем ее интервалам, скажем $\widetilde{\lambda}_{m}$, определим собственные значения, заключенные в интервалы данной группы, равными $\widetilde{\lambda}_{m}$.

В силу того, что таких групп $\lceil(n-r-2)+/(r+1)\rceil$, количество различных собственных значений равно $2+\lceil(n-r-2)+/(r+1)\rceil$. Теорема доказана.

\section{2. Задача модификации.}

ТЕорема 6. Минимальное число различных собственных значений среди задаваемых $\widehat{\lambda}_{1}, \ldots, \widehat{\lambda}_{n}$ в задаче модификации не мохсет быть меньше

$$
\left\lceil\frac{n}{2 r+1}\right\rceil \text {. }
$$

ДоказАтЕЛьСтво. Обозначим через $\Omega_{i}$ отрезок вида $\left[\lambda_{i}, \lambda_{i+1}\right], i=1, \ldots, n-1$. Из условий теоремы 4 следует, что числа $\widehat{\lambda}_{1}, \ldots, \widehat{\lambda}_{n}$ должны подчиняться условиям

$$
\begin{cases}\hat{\lambda}_{j} \leqslant \lambda_{j+r}, & j=1, \ldots, r, \\ \lambda_{j-r} \leqslant \widehat{\lambda}_{j} \leqslant \lambda_{j+r}, & j=r+1, \ldots, n-r, \\ \lambda_{j-r} \leqslant \widehat{\lambda}_{j}, & j=n-r+1, \ldots, n .\end{cases}
$$

То есть каждое $\widehat{\lambda}_{j}$ должно лишь содержаться в определенном интервале $G_{j}$, состоящем не более чем из $2 r+1$ отрезков $\Omega_{k}$ с последовательными номерами. Максимальное количество отрезков $G_{j}$, имеющих общую точку, равно $2 r+1$. Разобъем весь набор выбираемых $\widehat{\lambda}_{1}, \ldots, \widehat{\lambda}_{n}$ на групшы по $2 r+1$ с последовательными номерами (в последней может быть меньше). Обозначим для каждой группы $k$ точку пересечения соответствуюших интервалов $G_{j}$ через $\widetilde{\lambda}_{k}$ и положим $\widehat{\lambda}_{j}=\widetilde{\lambda}_{k}$ для всех $\widehat{\lambda}_{j}$ из группы $k$. Так как в каждой групше все задаваемые числа равны и числа $\widetilde{\lambda}_{k}$ попарно различны, то число различных собственных значений модифицированной матрищы не может быть меньше числа групп, а именно

$$
\left\lceil\frac{n}{2 r+1}\right\rceil \text {. }
$$

Теорема доказана.

\section{СПИСОК ЦИТИРОВАННОЙ ЛИТЕРАТУРЫ}

[1] Годунов С. К. Современные аспекты линейной алгебры. Новосибирск: Научная книга, 1997.

[2] Хорн Р., Джонсон Ч. Матричный анализ. М.: Мир, 1989.

Институт вычислительной математики РАН, г. Москва 